\title{
Diagnostic efficacy of rapid assays used for the detection of hepatitis B virus surface antigen
}

\author{
EWS Chameera ${ }^{1}, \mathrm{~F}_{\text {Noordeen }}{ }^{1}, \mathrm{H}_{\text {Pandithasundara }}{ }^{1}$, AMSB Abeykoon ${ }^{1}$ \\ Sri Lankan Journal of Infectious Diseases 2013 Vol.3(2):21-27 \\ DOI: http://dx.doi.org/10.4038/sljid.v3i2.5172
}

Key words: Rapid immunochromatography assays; Hepatitis B virus; Serum HBsAg; Enzyme immunoassays

\begin{abstract}
Objectives: Two rapid tests, CORTEZ'S HBSAg one step and CTK Biotech's Onsite HBsAg, for the detection of HBsAg in the serum were compared using the SURASE B-96 (TMB) enzyme immunoassay (EIA) as the comparator.
\end{abstract}

Methods: Fifty blood samples $(n=50)$ were tested using the two rapid tests and the EIA.

Results: Based on the EIA, 5 sera were positive for HBsAg and 45 sera were negative for HBsAg. However, 4 sera were positive while 46 sera were negative for HBsAg when tested by Biotech's Onsite HBsAg. Three (n=3) sera were positive while 47 sera were negative for $\mathrm{HBs} A g$ when tested by CORTEZ'S HBsAg one step detection kit. The specificity and the positive predictive value (PPV) were $100 \%$ for both rapid tests. However, the sensitivity and negative predictive value (NPV) for Biotech's Onsite HBsAg assay and CORTEZ'S HBsAg one step assay were $80 \%$ and $97.82 \%$ and $60 \%$ and $95.74 \%$, respectively.

Conclusions: Both rapid tests showed less sensitivity and NPV than the EIA. Sensitivity and NPV were higher withOnsite $H B s A g$ than the CORTEZ'S HBSAg making the former slightly better than the latter for detecting serum HBsAg. According to our findings, the accuracy of rapid tests for HBsAg detection varied compared to EIA, suggesting the importance of validating rapid tests routinely when used in diagnostic laboratories.

\section{Introduction}

Hepatitis B virus (HBV) infection is one of the major public health problems in the world. According to the World Health Organization (WHO), two billion people have been infected with $\mathrm{HBV}$ at some point in their life and more than 240 million individuals have become chronic carriers in whom 0.6 million deathsoccur each year. ${ }^{1}$ In adults, acute HBV infection causes fulminant lethal hepatitis in a very small percentage of patients andin most instances HBV infection will cause a mild disease with complete recovery and elimination of the virus from the

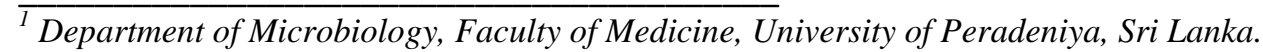

Address for correspondence: Dr. F. Noordeen, Department of Microbiology, Faculty of Medicine, University of Peradeniya, Sri Lanka.E mail:faseehan@pdn.ac.lk; faseeha.noordeen12@ gmail.com Fax:94- 81 2389106; Telephone:94- 812396532 
blood. ${ }^{2}$ However, in $5-10 \%$ of adult patients, the HBV infection will progress to chronic hepatitis B (CHB) which can lead to cirrhosis and hepatocellular carcinoma (HCC) which are life threatening. ${ }^{2}$ In contrast, in children, $90 \% \mathrm{HBV}$ infection will progress to $\mathrm{CHB}$ and due to immune tolerance these children will not have active hepatitis at the early phase of infection. However, those who get infected with HBV early in life will have the consequences of CHB when they age. Thus individuals with CHB will develop cirrhosis and HCC in their 60's or 70's due to slow destruction and transformation of the hepatocytes. ${ }^{4}$ Generally HBV itself does not damage hepatocytes but the immune attack mediated by cytotoxic $\mathrm{T}$ cells against the HBV antigen bearing hepatocytes causes the inflammation and necrosis. ${ }^{4}$

HBV is a DNA virus belonging to the Hepadnaviridae family. The envelope of the virus surrounds the nucleo-capsid which comprises the DNA genome and the viral matrix. HBV is transmitted through contact with blood and body fluids. ${ }^{3}$ Unlike human immunodeficiency virus (HIV), HBV can survive for at least seven days outside the body which makes HBV more infectious than HIV. ${ }^{1}$ In addition, HBV infection is silent in those infected at an early stage of their life. It has been observed that $80 \%$ of adults with CHB have no reason to seek medical help (including investigations) to know that they have been infected. ${ }^{5}$ Thus, accurate diagnosis of $\mathrm{CHB}$ or HBV infection by screening suspected populations or selected groups prior to elective invasive procedures such as gastroscopy or renal dialysis is routine practice. Conversely, screening for HBV infection during emergencies such as needle prick injuries and emergency blood transfusions is also important to minimize the spread of HBV.

Although several viral markers are available for the detection of $\mathrm{HBV}$, Hepatitis B surface antigen (HBsAg) is the major viral marker used for the detection of $\mathrm{HBV}$ infection. ${ }^{3}$ Acute HBV infection has an incubation period of $4-12$ weeks. ${ }^{2} \mathrm{HBsAg}$ is the first marker to appear and becomes detectable during this period. Usually HBsAg is evident in the non-specific prodrome and in the early phase of the acute infection and becomes undetectable in convalescence. The persistence of HBsAg for more than 6 months indicates CHB. ${ }^{4}$ Hence, HBsAg is used as the viral marker for both screening of a community as well as to diagnose a suspected acute HBV infection or CHB.

There are several immunological methods available to detect $\mathrm{HBsAg}$, including enzyme immunoassays (EIA), radioimmunoassays (RIA), immunochromatographic assays (ICA) and haemagglutination assays. Of these, EIAs and RIAs are the most sensitive methods. ${ }^{6,7}$ EIA methods are generally used by reference laboratories and blood banks because of its accuracy, low cost and safety when compared to RIA methods.

Rapid diagnostic tests based on immunochromatographic principles are widely used in most developing countries, including Sri Lanka, for the detection of HBsAg. These methods are considerably cheaper than EIA methods. Almost all ICAs can generate results within 30 minutes, therefore being less time consuming when compared to EIA. Another interesting feature of ICAs compared to EIA is that expert training is not required to perform an ICA. However, the question is, are the ICAs accurate enough to detect an acute $\mathrm{HBV}$ infection or $\mathrm{CHB}$, as both have serious consequences in HBV infection control in a particular setting or in the community.

Many studies have been performed to determine the accuracy indices of ICA based rapid tests on HBsAg detection. Different opinions have been forwarded by researchers and it has been difficult to come to a conclusion. An important feature is that different ICAs have shown different accuracy levels, although these assays are based on the same principle. ${ }^{5,7}$ A Japanese study has observed $100 \%$ sensitivity and specificity for an ICA called Dainascreen to detect 
HBsAg. ${ }^{6}$ In contrast to the Japanese study, Khan $e t a l^{8}$ has shown that ICAs are not reliable and should be backed up by superior methods such as EIA and PCR for detecting HBV infection.Accuracy of the results does not always depend on the product used for the tests which may also differ based on the population tested or the circulating HBV type in a particular location. Up to now, 8 different genotypes and 9 different serological subtypes of HBV have been discovered. ${ }^{5,9}$ Composition of these variant forms can be diverse in different countries. For instance, Manamperi et al (2012) have observed that HBV genotypes A, B,C,D and E circulate in Sri Lanka with mixed infections of these genotypes in some individuals tested in their study. ${ }^{10}$ Researchers have observed that all ICAs do not possess equal ability to detect all these HBV sub groups, which means some products have less ability to detectHBsAg from a certain HBV sub type. 5

Although rapid tests are widely used in Sri Lanka, studies on accuracy indices of ICAs in the country are scarce. It is not safe to depend on the studies that have been performed in other countries because genetic diversities in HBV can result in differences in accuracy indices for detecting HBsAg for a given ICA based rapid test. Hence, the current study was planned to compare two rapid ICA based tests that have been used widely in Sri Lanka for serum HBsAg detection, as test accuracy indices may vary among populations depending on the prevalence of HBV infection and the prevalent genotype of HBV of a given population.CORTEZ'S HBsAg one step detection kit (Diagnostic Automation/ Cortez Diagnostics Inc, USA) and CTK Biotech's Onsite HBsAg rapid test (CTK Biotech Inc, USA) with a standard commercial EIA SURASE B-96 (TMB) (General Biologicals Corp, Taiwan) were used to study the accuracy indices for detecting HBsAg in the sera of a selected group of individuals.

\section{Methods and Materials}

The selection of samples for the study was done using convenient sampling. Fifty patients' blood samples $(n=50)$ received by the Department of Microbiology, Faculty of Medicine, University of Peradeniya for detecting HBsAg between January and August 2010 were used for the study. These patients were mainly referred from the Surgical Unit of the Teaching Hospital, Peradeniya and other hospitals in the Kandy district. Three (3) $\mathrm{ml}$ of blood collected from each patient was allowed to clot at room temperature for 20 minutes. Serum was separated by centrifuging at 4000 rpm for 10 minutes and stored in cryovials at $-20^{\circ} \mathrm{C}$ until tested.

Each serum sample was tested using CORTEZ'S HBsAg one step detection kit, CTK Biotech's Onsite HBsAg rapid test and the SURASE B-96 (TMB) EIA. The sera were allowed to thaw just prior to testing and tests performed after bringing the sera to the room temperature. All the tests were performed on the same dayfollowing the manufactures instructions.

\section{CORTEZ'S HBsAg one step rapid test}

This is a rapid, direct binding test for the visual detection of HBsAg in serum/plasma which is based on the principle of sandwich immunoassay. Monoclonal and polyclonal antibodies are employed to identify HBsAg specifically. The results were read 10-20 minutes after placing 4 drops $(120 \mu \mathrm{l})$ of serum in the sample well.

\section{Onsite HBsAg rapid test}

This test is a lateral flow chromatographic immunoassay. The test cassette consists of a burgundy coloured conjugate pad containing mouse anti-HBsAg antibody conjugated with colloid gold and a nitrocellulose membrane strip containing a test band ( $\mathrm{T}$ band) and a control band ( $\mathrm{C}$ band). The $\mathrm{T}$ band is pre-coated with non-conjugated HBsAg antibody and the $\mathrm{C}$ band is pre-coated with 
goat anti-mouse IgG antibody. HBsAg if present in the specimen would bind to the $\mathrm{HBs} \mathrm{Ag} / \mathrm{Ab}$ conjugates. The immune-complex is then captured on the membrane by the pre-coated nonconjugated HBsAg antibody. The results were read 15 minutes after adding 3 drops $(90 \mu \mathrm{L})$ of serum to the sample well.

\section{SURASE B-96 (TMB) EIA}

The solid phase of the microtiter plate is made of polystyrene wells coated with mouse monoclonal antibodies specific for HBsAg whereas guinea pig polyclonal antibody purified by affinity chromatography is used to prepare the anti-HBs peroxidase conjugate. When serum/plasma containing HBsAg is added to wells together with the peroxidase conjugated anti HBs antibody and incubated, an antibody-HBsAg-antibody-peroxidase complex will form on the wells. After the solution of TMB substrate is added to the wells and incubated, a colour develops in proportion to the amount of HBsAg bound to anti-HBs. After addition of sulfuric acid, the plate was read at $450 \mathrm{~nm}$ with a selected reference wavelength within 620 to $690 \mathrm{~nm}$. Positive results were confirmed by retesting in duplicate.

\section{Results}

Sensitivity, specificity, positive predictive value (PPV) and negative predictive value (NPV) for HBsAg detection were calculated for Onsite HBsAg Rapid test and CORTEZ'S HBsAg One Step test using SURASE B-96 (TMB) EIA as the comparator (Sensitivity $=100 \%$; specificity $=$ 99.95\%).

Of the 50 samples tested according to SURASE B-96 (TMB) EIA, 45 serum samples were negative and 5 serum samples were positive for the presence of HBsAg.All the samples were tested in duplicate to ensure the validity of the results (Table 1).

Table 1: HBsAg detection using the EIA and 2 rapid assays

\begin{tabular}{llll}
\hline Assays for HBsAg detection & $\begin{array}{l}\text { HBsAg } \\
\text { Negative }\end{array}$ & $\begin{array}{l}\text { HBsAg } \\
\text { Positives }\end{array}$ & Total \\
\hline SURASE B-96 EIA (comparator) & 45 & 05 & 50 \\
Onsite HBsAg rapid assay & 46 & 04 & 50 \\
CORTEZ'S HBsAg one step assay & 47 & 03 & 50 \\
\hline
\end{tabular}

Of the 50 serum samples tested using the Onsite HBsAg Rapid assay, 46 were negative and 4 positive for the presence of HBsAg. Of the 50 serum samples tested using CORTEZ'S HBsAg one step detection assay, 47 were negative and 3 positive for the presence of HBsAg (Table 1). The accuracy indices, including sensitivity, specificity, PPV and NPV were calculated using the SURASE B-96 ELISA test as the standard (Table 2).

Table 2: Accuracy indices of the rapid assays

\begin{tabular}{lllll}
\hline Accuracy indices & Sensitivity & Specificity & PPV & NPV \\
\hline Onsite HBsAg rapid assay & $80 \%$ & $100 \%$ & $100 \%$ & $97.8 \%$ \\
CORTEZ'S HBsAg one step assay & $60 \%$ & $100 \%$ & $100 \%$ & $95.7 \%$ \\
\hline
\end{tabular}

Comparator :SURASE B-96 EIA 


\section{Discussion}

For a highly infectious virus like HBV which causes a long term silent infection, accurate detection of the viral marker is essential for controlling the transmission of the virus. For this reason, it is necessary to validate detection methods prior to allowing their use in diagnostic laboratories. Moreover, performing routine validations at regular intervals while a test is in use will allow evaluation of that test in a particular laboratory. In many developing countries, ICA based rapid diagnostic tests are widely used to detect HBsAg for both diagnosis and screening of acute and chronic HBV infections, although ideally, screening should be done using more advanced and accurate methods such asEIA or ELISA. Negative samples from patients referred for screening assays (rapid assays) are seldom re-tested, considering the costs of re-testing in resource poor settings. Hence, choosing a test with high sensitivity and NPV is more important than choosing a test with high specificity and PPV for routine use.

Results of the current study indicated that both ICAs tested are less accurate when compared to the EIA. Specificity and the PPV were $100 \%$ for both ICAs. However, the sensitivity and the NPV were less when compared to the EIA. Although discrepancies between the two ICA based rapid assays compared with the EIA are seen, due to the small sample size, definitive conclusions cannot be reached and further evaluation using a larger sample are recommended. Similarly, although sensitivity and NPV were higher using the Onsite HBsAg rapid assay than the CORTEZ'S HBsAg one step detection assay (Table 2), comparison using a larger sample would be needed before a definite recommendation can be made.

Some studies have observed ICAs to have high sensitivity and specificity. ${ }^{7,6,11}$ A study reported by Ansari et al (2007) showed that rapid assays with strip or device had sensitivity between 97.5 to $99.2 \%$ and specificity between 97.5 and $99.2 \%{ }^{7}$ In a different study using two ICAs, the sensitivity and specificity were $100 \%$. $^{6}$ According to Lin et al (2008), DRW-HBsAg rapid test, an ICA based rapid assay, demonstrated an overall specificity of $98.7 \%$ and its sensitivity was almost $100 \% .{ }^{11}$ In contrast, several evaluation studies have noted that the specificity and the PPV are high in ICAs but sensitivity and the NPV are less as observed in our study. Kauret al. (2000) has observed that rapid assay used for HBsAg detection in their study (Hepacard, India) has a specificity of $100 \%$ but the sensitivity was $93.4 \% .^{12}$ In a different study in India, the Hepacard assay was found to have a sensitivity of $79 \%$ and specificity of $98.9 \% .{ }^{13}$ Khan et al. (2010) using two ICA based rapid assays for HBsAg detection found sensitivity to be $53 \%$ and $50 \%$, respectively although the specificity was $100 \%$ and $95 \%$ respectively. ${ }^{8}$

Different ICA based rapid assays used for HBsAg detection in the serum may not have the same accuracy index in every region since there can be differences in the prevalence of HBV infection in a given population. Moreover, the circulating subtype/s of HBV in a locale can also be different. In such cases ICA that does not cover this particular subtype/s will not detect this type when testing. This may be the reason why one serum sample that was non-reactive for both Onsite HBsAg rapid and CORTEZ'S HBsAg one step testwas reactive using the EIA. Further work is needed as data on the circulating genotypes and mutants of HBV are not widely available in Sri Lanka.

There are no approved rapid assays by the food and drug administration (FDA) and CE mark for European Union for HBsAg detection although several rapid tests for screening for HIV have been approved. ${ }^{11}$ Taking all these findings into account, we can suggest that the ICAs need regular validation with an accepted EIA for the detection of HBsAg if rapid ICAs are used for diagnostic and screening purposes in resource poor settings.Rapid assays must be used with 
caution and it is also important to validate these rapid assays by testing them in a given population to assess the effectiveness of these assays in detecting the genotypes and subtypes of HBV circulating in the region before using these testsroutinely in diagnostic laboratories.

In conclusion, based on our findings, both ICAs tested were less accurate when compared to the EIA. Specificity and the PPV were $100 \%$ for both ICAs. However, the sensitivity and the NPV were less when compared to the EIA. Sensitivity and NPV were higher for the Onsite HBsAg rapid assay than the CORTEZ'S HBsAg one step assay for detecting HBsAg, making the Onsite HBsAg rapid assay better than the CORTEZ'S HBsAg assay for the detection of HBsAg in serum or plasma.Further work with increased sample size is recommended for evaluation of these commonly used test kits

\section{Acknowledgements}

Funding from annual allocation to the Department of Microbiology, Faculty of Medicine, University of Peradeniya, Sri Lanka.

\section{Conflict of interest}

None declared by the authors.

\section{Ethics}

The study used blood samples received by the Microbiology Laboratory for HBsAg testing. Verbal consent was obtained from the study cohort that the HBsAg testing data from a set of samples would be analyzed to determine the accuracy indices of rapid immunochromatography assays on HBsAg detection using a standard ELISA.

\section{References}

1. World health organization. Hepatitis B. WHO media center online 2012; 8:10. URL:http://www.who.int/mediacentre/factsheets/fs204/en/

2. Kayser FH, Bien KA, Eckert J, Zinkernagel RM.Medical Microbiology 2005. $10^{\text {th }}$ ed. New York.

3. Lee JM,Ahn SH.Quantification of HBsAg: Basic virology for clinical practice. World Journal of Gastroenterology 2011;17:283-89.http://dx.doi.org/10.3748/wjg.v17.i3.283

4. Levinson W. Review of Medical Microbiology and Immunology 2008. $10^{\text {th }}$ ed. USA.

5. ScheiblauerH, El-Nagesh M, Diaz S, Nick S, Zeichhardt H, Grunert HP et al. Performance evaluation of 70 hepatitis B virus surface antigen assays from around the world by a geographically diverse panel with an array of HBV genotypes and HBsAg subtypes. International Journal of Transfusion Medicine 2009; 98:403-14. doi: 10.1111/j.1423-0410.2009.01272.x

6. Sato K, Ichiyama S, Iinuma Y, Nada T, Shimokata K, Nakashima N. Evaluation of immunochromatographic assay systems for rapid detection of hepatitis B surface antigen and antibody, Dainascreen HBsAg and DainascreenAusab. Journal of Clinical Microbiology 1996; 34:1420-22.No doi

7. Ansari MHK, Omrani MD, MovahediV. Comparative evaluation of immunochromatographic rapid diagnostic tests and PCR methods for detection of human hepatitis B surface antigen. Hepatitis monthly 2007; 7:87-91 No doi. 
8. Khan JK, Lone DS, HameedA, Munim MR, Bhatti M, Khattak AA et al. Evaluation of the performance of two rapid immunochromatographic tests for detection of HBsAg and anti HCV antibody using ELISA tested samples. Annals of King Edward Medical University 2010; 16(S1):84-7.No doi

9. Purdy MA, Talekar G, Swenson P, Aufra A, Fields H. A new algorithm for deduction of hepatitis B surface antigen subtype determinants from the amino acid sequence. Intervirology 2006; 50:45-51 http://dx.doi.org/10.1159/000096312

10. Manamperi A, Gunawardene NS, Wellawatta C, Abewickreme W, De Silva HJ. Hepatitis B virus genotypes in a group of Sri Lankan patients with chronic infection. Tropical Biomedicine 2011;28:320-24. No doi

11. Lin Y, Wang Y, Loua A, Day G, Qiu Y, NadalaJr ECB et al. Evaluation of a new hepatitis B virus surface antigen rapid test with improved sensitivity. Journal of Clinical Microbiology 2008; 46:3319-24 http://dx.doi.org/10.1128/JCM.00498-08

12. Kaur H, Dhanao J, Oberoi A. Evaluation of rapid kits for detection of HIV, HBsAg and HCV infections. Indian Journal of Medical Science 2000;54:432-4 http://www.indianjmedsci.org/text.asp?2000/54/10/432/12122

13. Raj AA, Subramaniam T,Raghuraman S, Abraham P. Evaluation of an indigenously manufactured rapid immunochromatographic test for detection of HBsAg. Indian Journal of Pathology and Microbiology 2001; 44:413-4. No doi

14. Mvere D, Constantine NT, Katsawde E, Tobaiwa O, Dambire S, Corcoran P. Rapid and simple hepatitis assays: Encouraging results from a blood donor population in Zimbabwe. Bulletin of World Health Organization 1996; 74:19-24. No doi 